\title{
What Happened to Fibrinolysis?
}

\author{
Victor Gurewich* \\ Department of Medicine, Harvard Medical School, Mount Auburn Hospital, USA
}

*Corresponding author: Victor Gurewich, Department of Medicine, Harvard

Medical School, Mount Auburn Hospital, USA.

Received Date: August 23, 2021

Published Date: September 09, 2021

\section{Mini Review}

Fibrinolysis refers to the natural enzymatic system responsible for dissolving a blood clot or thrombus. Since an intravascular thrombus is responsible for almost all heart attacks and most strokes, interest in fibrinolysis, the only medical treatment, dominated medical interest in the 60's-90's. Since that time, the incidence of heart attacks and strokes has not changed much, but interest in fibrinolysis has faded. Instead, interest has shifted to catheter removal of clots, called percutaneous coronary intervention ("PCI") which is a complicated procedure that is slower, and much more costly than fibrinolysis. It is also a cruder treatment that can only remove clots larger than the catheter, but it is handsomely reimbursed. By contrast, fibrinolysis is a much faster, cheaper, treatment which is not limited to larger vessels, so what happened to it?

The fault is both intellectual, related to a misunderstanding of fibrinolysis and financial or profit-motivated. The latter is the easiest to identify. The current treatment of a heart attack or stroke is PCI, which is well-compensated and an important revenue source for cardiology departments and hospitals. It has become the dominant treatment also because fibrinolysis was conveniently misunderstood. For more than three decades, fibrinolysis has meant the administering a single fibrinolytic, usually tissue plasminogen activator (tPA), which is known to be a weak fibrinolytic. More importantly, it has been recognized since 1984, when prouPA was discovered that fibrinolysis requires two fibrinolytics, tPA and prouPA, which is much more effective than and tPA alone. In fact, prouPA (which has two forms), is responsible for two-thirds of fibrinolysis and tPA one third, which explains why tPA is such a weak fibrinolytic.

These findings describing the presence and importance of the second activator were ignored, and fibrinolysis continued to mean giving tPA alone. This meant deliberately handicapping fibrinolysis which was done in order to find an excuse to change reperfusion treatment of heart attack and, when possible, stroke to a catheter, percutaneous coronary intervention (PCI). This is a more financially rewarding treatment, but it also denies the natural system of dissolving blood clots, which requires two fibrinolytics. By undermining fibrinolysis at left PCI to become the treatment of choice for heart attacks and some strokes, and is treatment was motivated by its generous reimbursement than by its efficacy. The evidence for this is that PCI to date has never been compared with full fibrinolysis, only with tPA fibrinolysis which is only one third as effective as full fibrinolysis which requires both tPA and prouPA. Comparing PCI with fully effective fibrinolysis has so far been avoided, leaving PCI as the treatment of choice, and a major source of revenue.

Fibrinolysis by the tPA and uPA combination, which promises to be the most effective method to remove an obstructive intravascular blood clot has been successfully ignored, and fibrinolysis with tPA alone has been retained providing an an excuse for catheter intervention. This remains the order of the day for financial reasons only. The catheter brings in more money but is less effective and is associated with a small risk, whereas the evidence is that fibrinolysis with both TPA and prouPA is the most effective treatment of heart attack and stroke and carries little to no risk, since very little tPA is required.

\section{Acknowledgement}

None.

\section{Conflict of Interest}

No conflict of interest. 